\title{
BIBO Stabilization of Discrete-Time Stochastic Control Systems with Mixed Delays and Nonlinear Perturbations
}

\author{
Xia Zhou, ${ }^{1}$ Yong Ren, ${ }^{2}$ and Shouming Zhong ${ }^{3}$ \\ ${ }^{1}$ School of Mathematics and Computational Science, Fuyang Teachers College, Fuyang 236037, China \\ ${ }^{2}$ Department of Mathematics, Anhui Normal University, Wuhu 241000, China \\ ${ }^{3}$ College of Applied Mathematics, University of Electronic Science and Technology of China, Chengdu, Sichuan 611731, China
}

Correspondence should be addressed to Xia Zhou; zhouxia44185@163.com

Received 19 March 2013; Revised 23 May 2013; Accepted 6 June 2013

Academic Editor: Sakthivel Rathinasamy

Copyright (C) 2013 Xia Zhou et al. This is an open access article distributed under the Creative Commons Attribution License, which permits unrestricted use, distribution, and reproduction in any medium, provided the original work is properly cited.

\begin{abstract}
The problem of bounded-input bounded-output (BIBO) stabilization in mean square for a class of discrete-time stochastic control systems with mixed time-varying delays and nonlinear perturbations is investigated. Some novel delay-dependent stability conditions for the previously mentioned system are established by constructing a novel Lyapunov-Krasovskii function. These conditions are expressed in the forms of linear matrix inequalities (LMIs), whose feasibility can be easily checked by using MATLAB LMI Toolbox. Finally, a numerical example is given to illustrate the validity of the obtained results.
\end{abstract}

\section{Introduction}

Many dynamical systems not only depend on the present states but also involve the past ones, generally called the timedelay systems. Generally, as a source of poor or significantly deteriorated performance and instability for the concerned closed-loop system, the time delays are unavoidable in technology and nature. Many works have been done on the stability of time-delay systems; one can see [1-23] and the references therein. The dynamics analysis of continuoustime systems with distributed delay has been well studied in $[9-12,20]$. The aspect of simulation and application in control systems, whereas, discrete-time control systems play a more important role than their continuous-time counterparts in the practical digital world. If one wants to simulate or compute the continuous-time systems, it is essential to formulate the discrete-time analogue so as to investigate the dynamical characteristics. It is necessary to take continuous distributed delays into account for modeling realistic systems, for example, neural networks; due to the presence of an amount of parallel pathways of a variety of axon sizes and lengths, a neural network usually has a spatial nature. Very recently, Liu et al. introduced the infinite distributed delay and distributed delay in the form of constant delay into the delay neural networks. See [17-19].
In order to track out the reference input signal in real world, the bounded-input bounded-output stabilization has been investigated by many researchers, one can see [20$32]$ and the references therein. In $[22,23]$, the sufficient conditions for BIBO stabilization of control systems with no delays were proposed by the Bihari type inequality. In $[9,10]$, by employing the parameters technique and the Gronwall inequality, the authors investigated the BIBO stability of the systems without distributed time delays. In [20, 27, 29], based on Riccati equations and by constructing appropriate Lyapunov functions, some BIBO stabilization conditions for a class of delayed control systems with nonlinear perturbations were established. In [30], the BIBO stabilization problem of a class of piecewise switched linear systems was further investigated. It should be pointed out that almost all results concerning the BIBO stability for control systems mainly concentrate on continuous-time models. Seldom works have been done for discrete-time control systems one can see [21, 28]. In addition, the previously mentioned works just considered the deterministic systems (see, e.g., [31, 32]). The deterministic systems often fluctuate due to noise, which is random or at least appears to be so. Therefore, we must move from deterministic problems to stochastic ones. So, the BIBO stabilization for stochastic control systems case is necessary and interesting. To the best of our knowledge, there is no 
work reported on the mean square BIBO stabilization for the discrete-time stochastic control systems with mixed timevarying delays.

It is well known that the classical technique applied in the study of stability is based on the Lyapunov direct method. However, the Lyapunov direct method has some difficulties with the theory and application to specific problems while discussing the stability of solutions in stochastic systems with time delay. In [33], the midpoint in the time delay's variation interval is introduced, and the variation interval is divided into two subintervals with equal length, by constructing the Lyapunov functional which involved midpoint to reduce the conservatism of stability conditions. This method was first proposed to study the stability and stabilization problems for linear continuous-time systems, and then many successful applications were found in [13-15]. In this paper, we will reconsider this method by introducing a new piecewiselike delay method, given that the point of the time delay's variation interval is arbitrary point rather than midpoint.

Motivated by the aforementioned works, in this paper, we investigate BIBO stabilization in mean square for a class of discrete-time stochastic control systems with mixed timevarying delays and nonlinear perturbations. Some novel delay-dependent stability conditions for the previously mentioned system are derived by constructing a novel LyapunovKrasovskii function. These conditions are expressed in the forms of linear matrix inequalities, whose feasibility can be easily checked by using MATLAB LMI Toolbox. Finally, a numerical example is given to illustrate the validity of the obtained results.

The paper is organized as follows. In Section 2, some notations and the problem formulation are proposed. The main results are given in Section 3. In Section 4, a numerical example is given to illustrate the validity of the obtained theory results. The conclusion is proposed in Section 5 .

\section{Notations and Problem Formulation}

Firstly, we propose some notations which will be needed in the sequel. The notations are quite standard. Let $R^{n}$ and $R^{n \times m}$ denote, respectively, the $n$-dimensioned Euclidean space and the set of all $n \times m$ real matrices. The superscript " $T$ " denotes the transpose and the notation $X \geq Y$ (respective $X>Y$ ) means that $X$ and $Y$ are symmetric matrices and that $X-Y$ is positive semidefinitive (respective positive definite). Let $\|\cdot\|$ denote the Euclidean norm in $R^{n}$, let $N^{+}$denote the positive integer set, and let $I$ be the identity matrix with compatible dimension. If $A$ is a matrix, denote by $\|A\|$ its operator norm; that is, $\|A\|=\sup \{\|A x\|:\|x\|=1\}=\sqrt{\lambda_{\max }\left(A^{T} A\right)}$, where $\lambda_{\text {max }}(A)$ (resp., $\left.\lambda_{\text {min }}(A)\right)$ means the largest (resp., smallest) value of $A$. Moreover, let $\left(\Omega, \mathscr{F},\left\{\mathscr{F}_{t}\right\}_{t \geq 0}, P\right)$ be a complete probability space with a filtration $\left\{\mathscr{F}_{t}\right\}_{t \geq 0}$ satisfying the usual conditions (i.e., the filtration contains all $P$-null sets and is right continuous). $E\{\cdot\}$ stands for the mathematical expectation operator with respect to the given probability measure $P$. The asterisk $*$ in a matrix is used to denote term that is induced by its symmetry. Matrices, if not explicitly state are assumed to have compatible dimensions. Denote
$N[a, b]:=\{a, a+1, \ldots, b\}$. Sometimes, the arguments of the functions will be omitted in the analysis without confusions.

In this paper, we consider the discrete-time stochastic control system with mixed time-varying delays and nonlinear perturbations with the following form:

$$
\begin{aligned}
& x(k+1)= A x(k)+B x(k-\tau(k))+C u(k) \\
&+D \sum_{i=-d(k)}^{-1} h(x(k+i))+f(k, x(k), x(k-\tau(k))) \\
&+(G x(k)+H x(k-d(k))) \omega(k), \\
& y(k)=M x(k)
\end{aligned}
$$

where $x(k)=\left[x_{1}(k), x_{2}(k), \ldots, x_{n}(k)\right]^{T} \in R^{n}$ denotes the state vector, $u(k)=\left[u_{1}(k), u_{2}(k), \ldots, u_{m}(k)\right]^{T} \in R^{m}$ is the control input vector, $y(k)=\left[y_{1}(k), y_{2}(k), \ldots, y_{n}(k)\right]^{T} \in R^{n}$ is the control output vector, $h(x(k))=\left[h_{1}(x(k)), h_{2}(x(k)), \ldots\right.$, $\left.h_{n}(x(k))\right]^{T}, A, B, D, G, C, H$, and $M$ represent the weighting matrices with appropriate dimension, and the positive integers $\tau(k)$ and $d(k)$ are the discrete-time-varying delay, distributed time-varying delay and respectively, satisfying that

$$
\tau_{1} \leq \tau(k) \leq \tau_{2}, \quad d_{1} \leq d(k) \leq d_{2}, \quad k \in N^{+},
$$

with $\tau_{1}, \tau_{2}, d_{1}$, and $d_{2}$ being four known positive integers. For any given $\tau^{*} \in\left(\tau_{1}, \tau_{2}\right), d^{*} \in\left(d_{1}, d_{2}\right)$. The initial conditions of the system (1) are given by

$$
x(k)=\phi(k), \quad k \in\left[-\max \left\{\tau_{2}, d_{2}\right\}, 0\right] .
$$

The nonlinear vector-valued perturbation $f(k, x(k), x(k-$ $\tau(k)))$ satisfies that

$$
\begin{aligned}
& \|f(k, x(k), x(k-\tau(k)))\|^{2} \\
& \quad \leq \alpha_{1}\|x(k)\|^{2}+\alpha_{2}\|x(k-\tau(k))\|^{2},
\end{aligned}
$$

where $\alpha_{1}$ and $\alpha_{2}$ are two positive constants. $\omega(k)$ is a scalar Wiener process defined on $\left(\Omega, \mathscr{F},\left\{\mathscr{F}_{t}\right\}_{t \geq 0}, P\right)$ with

$$
\begin{aligned}
& E(\omega(k))=0, \quad E\left(\omega(k)^{2}\right)=1, \\
& E(\omega(i) \omega(j))=0, \quad i \neq j .
\end{aligned}
$$

Remark 1 . The $\tau^{*}$ divides the discrete-time delay's variation interval into two subintervals, that is, $\left[\tau_{1}, \tau^{*}\right]$ and $\left(\tau^{*}, \tau_{2}\right]$, and $d^{*}$ divides the distributed time delay's variation interval into two subintervals, that is, $\left[d_{1}, d^{*}\right]$ and $\left(d^{*}, d_{2}\right]$. We will discuss the variation of differences of the Lyapunov-Krasovskii functional $V(t, x(t))$ for each subinterval. Compared with the previous results in the works of [27-32], the BIBO stability conditions are derived in this paper by checking the variation of $V(t, x(t))$ in subintervals rather than in the whole variation interval of the delays.

In what follows, we describe the controller with the form

$$
u(k)=K x(k)+r(k),
$$


where $K$ is the feedback gain matrix and $r(k)$ is the reference input.

Assumption 2. For any $\xi_{1}, \xi_{2} \in R, \xi_{1} \neq \xi_{2}$, let

$$
\gamma_{i}^{-} \leq \frac{h_{i}\left(\xi_{1}\right)-h_{i}\left(\xi_{2}\right)}{\xi_{1}-\xi_{2}} \leq \gamma_{i}^{+},
$$

where $\gamma_{i}^{-}$and $\gamma_{i}^{+}$are known constant scalars.

Remark 3. The constants $\gamma_{i}^{-}, \gamma_{i}^{+}$in Assumption 2 are allowed to be positive, negative, or zero. Hence, the function $h(x(k))$ could be nonmonotonic and is more general than the usual sigmoid functions and the recently commonly used Lipschitz conditions.

At the end of this section, let us introduce some important definitions and lemmas as which will be used in the sequel.

Definition 4 (see $[28,31])$. A vector function $r(k)=\left(r_{1}(k)\right.$, $\left.r_{2}(k), \ldots, r_{n}(k)\right)^{T}$ is said to be an element of $L_{\infty}^{n}$, if $\|r\|_{\infty}=$ $\sup _{k \in N[0, \infty)}\|r(k)\|<+\infty$, where $\|\cdot\|$ denotes the Euclid norm in $R^{n}$, or the norm of a matrix.

Definition 5 (see $[28,31]$ ). The nonlinear stochastic control system (1) is said to be BIBO stability in mean square, if we can construct a controller (6) such that the output $y(k)$ satisfies that

$$
\mathbb{E}\|y(k)\|^{2} \leq N_{1}+N_{2}\|r\|_{\infty}^{2}
$$

where $N_{1}$ and $N_{2}$ are two positive constants.

Lemma 6 (see [28]). For any given vectors $v_{i} \in R^{n}, i=1$, $2, \ldots, n$, the following inequality holds:

$$
\left[\sum_{i=1}^{n} v_{i}\right]^{T}\left[\sum_{i=1}^{n} v_{i}\right] \leq n \sum_{i=1}^{n} v_{i}^{T} v_{i} .
$$

Lemma 7 (see [28]). Let $x, y \in R^{n}$ and any $n \times n$ positivedefinite matrix $Q>0$. Then, one has

$$
2 x^{T} y \leq x^{T} Q^{-1} x+y^{T} Q y .
$$

Lemma 8 (see [28]). Given the constant matrices $\Omega_{1}, \Omega_{2}$, and $\Omega_{3}$ with appropriate dimensions, where $\Omega_{1}=\Omega_{1}^{T}$ and $\Omega_{2}=$ $\Omega_{2}^{T}>0$, then $\Omega_{1}+\Omega_{3}^{T} \Omega_{2}^{-1} \Omega_{3}<0$ if and only if

$$
\left(\begin{array}{cc}
\Omega_{1} & \Omega_{3}^{T} \\
* & -\Omega_{2}
\end{array}\right)<0 \quad \text { or } \quad\left(\begin{array}{cc}
-\Omega_{2} & \Omega_{3} \\
* & \Omega_{1}
\end{array}\right)<0 .
$$

\section{BIBO Stabilization for the System (1)}

In this section, we aim to establish our main results based on the LMI approach. For the conveniences, we denote

$$
\begin{aligned}
& \Gamma_{1}=\operatorname{diag}\left\{\gamma_{1}^{-} \gamma_{1}^{+}, \gamma_{2}^{-} \gamma_{2}^{+}, \ldots, \gamma_{n}^{-} \gamma_{n}^{+}\right\} \text {, } \\
& \Gamma_{3}=\operatorname{diag}\left\{\gamma_{1}^{+}, \gamma_{2}^{+}, \ldots, \gamma_{n}^{+}\right\} \text {, } \\
& \Gamma_{2}=\operatorname{diag}\left\{\frac{\gamma_{1}^{-}+\gamma_{1}^{+}}{2}, \frac{\gamma_{2}^{-}+\gamma_{2}^{+}}{2}, \ldots, \frac{\gamma_{n}^{-}+\gamma_{n}^{+}}{2}\right\} \text {, } \\
& a=\tau_{2}-\tau_{1}+1, \\
& b= \begin{cases}\frac{\left(d^{*}+d_{1}-1\right)\left(d^{*}-d_{1}\right)+2 d^{*}}{2}, & d_{1} \leq d(k) \leq d^{*} \\
\frac{\left(d^{*}+d_{2}-1\right)\left(d_{2}-d^{*}\right)+2 d_{2}}{2}, & d^{*}<d(k) \leq d_{2},\end{cases} \\
& c= \begin{cases}d^{*}, & d_{1} \leq d(k) \leq d^{*} \\
d_{2}, & d^{*}<d(k) \leq d_{2},\end{cases} \\
& \theta(k)= \begin{cases}x\left(k-\tau_{1}\right), & \tau_{1} \leq \tau(k) \leq \tau^{*} \\
x\left(k-\tau_{2}\right), & \tau^{*}<\tau(k) \leq \tau_{2},\end{cases} \\
& \tilde{\tau}= \begin{cases}\tau^{*}-\tau_{1}, & \tau_{1} \leq \tau(k) \leq \tau^{*} \\
\tau_{2}-\tau^{*}, & \tau^{*}<\tau(k) \leq \tau_{2},\end{cases} \\
& \beta= \begin{cases}\frac{\tau(k)-\tau_{1}}{\tau^{*}-\tau_{1}}, & \tau_{1} \leq \tau(k) \leq \tau^{*} \\
\frac{\tau_{2}-\tau(k)}{\tau_{2}-\tau^{*}}, & \tau^{*}<\tau(k) \leq \tau_{2} .\end{cases}
\end{aligned}
$$

Theorem 9. For given positive integers $\tau_{1}, \tau_{2}, d_{1}$, and $d_{2}$, under Assumption 2, the nonlinear discrete-time stochastic control system (1) with the controller (6) is BIBO stabilization in mean square, if there exist symmetric positive-definite matrix $P, R, Q_{i}, i=1,2, \ldots, 5, Z_{1}, Z_{2}$, and $X$ with appropriate dimensional, positive-definite diagonal matrices $\Lambda$ and some positive constants $\zeta$ and $\lambda^{*}$ such that the following two LMIs hold:

$$
P+2\left(\tau^{* 2} Z_{1}+\tilde{\tau}^{2} Z_{2}\right) \leq \lambda^{*} I
$$

$\Xi$

$$
=\left(\begin{array}{ccccccccc}
\Xi_{11} & \Xi_{12} & Z_{1} & 0 & 0 & \Gamma_{2} \Lambda & \Xi_{17} & \Xi_{18} & \sqrt{2} X \\
* & \Xi_{22} & \Xi_{23} & 0 & \Xi_{25} & 0 & B^{T} D & B^{T} & 0 \\
* & * & \Xi_{33} & 0 & 0 & 0 & 0 & 0 & 0 \\
* & * & * & -Q_{2} & 0 & 0 & 0 & 0 & 0 \\
* & * & * & * & \Xi_{55} & 0 & 0 & 0 & 0 \\
* & * & * & * & * & \Xi_{66} & 0 & 0 & 0 \\
* & * & * & * & * & * & \Xi_{77} & \lambda^{*} D^{T} & 0 \\
* & * & * & * & * & * & * & -\zeta I & 0 \\
* & * & * & * & * & * & * & * & -\lambda^{*} I
\end{array}\right)
$$

$\leq 0$ 
where

$$
\begin{aligned}
\Xi_{11}= & Q_{1}+Q_{2}+Q_{3}+a\left(Q_{3}+Q_{4}\right)-Z_{1} \\
& +Q_{5}-\Gamma_{1} \Lambda+G^{T} \lambda^{*} G+2 \tau^{* 2} Z_{1}+2 \widetilde{\tau}^{2} Z_{2} \\
& +2 \lambda^{*} \alpha_{1} I+\alpha_{1} \zeta I-P+2 \lambda^{*} A^{T} A-2 A^{T} X-2 X^{T} A, \\
\Xi_{12}= & \lambda^{*} A^{T} B-X^{T} B+G^{T} \lambda^{*} H, \\
\Xi_{17}= & \lambda^{*} A^{T} D-X^{T} D, \\
\Xi_{18}= & \lambda^{*} A^{T}-X^{T}, \\
\Xi_{22}= & H^{T} \lambda^{*} H+2 \lambda^{*} B^{T} B-Q_{4}-2 Z_{2}-\beta Z_{2} \\
& +2 \lambda^{*} \alpha_{2} I+\alpha \alpha_{2} \zeta-(1-\beta) Z_{2}, \\
\Xi_{23}= & Z_{2}+\beta Z_{2}, \\
\Xi_{25}= & Z_{2}+(1-\beta) Z_{2}, \\
\Xi_{33}= & -Q_{1}-Z_{1}-Z_{2}-\beta Z_{2}, \\
\Xi_{55}= & -Q_{5}-Z_{2}-(1-\beta) Z_{2}, \\
\Xi_{66}= & b R-\Lambda, \\
\Xi_{77}= & 2 \lambda^{*} D^{T} D-\frac{1}{c} R, \\
X= & -\lambda^{*} C K .
\end{aligned}
$$

Proof. We construct the following Lyapunov-Krasovskii function for the system (1):

$$
\begin{aligned}
V(k, x(k))= & V_{1}(k, x(k))+V_{2}(k)+V_{3}(k) \\
& +V_{4}(k)+V_{5}(k)+V_{6}(k),
\end{aligned}
$$

where

$$
\begin{aligned}
V_{1}(k, x(k))=x^{T}(k) P x(k), & \\
V_{2}(k)= & \sum_{i=k-\tau^{*}}^{k-1} x^{T}(i) Q_{1} x(i) \\
& +\sum_{i=k-d^{*}}^{k-1} x^{T}(i) Q_{2} x(i), \\
V_{3}(k)= & \sum_{i=k-\tau(k)}^{k-1} x^{T}(i) Q_{3} x(i)+\sum_{i=\tau_{1}}^{\tau_{2}-1} \sum_{j=k-i}^{k-1} x^{T}(j) Q_{3} x(j) \\
& +\sum_{i=k-\tau_{1}}^{k-1} x^{T}(i) Q_{3} x(i) \\
& +\sum_{i=-\tau_{2}+1}^{-\tau_{1}+1} \sum_{j=k-1+i}^{k-1} x^{T}(j) Q_{4} x(j), \\
V_{4}(k)= & \tau^{*} \sum_{i=-\tau^{*}}^{-1} \sum_{j=k+i}^{k-1} \eta^{T}(j) Z_{1} \eta(j),
\end{aligned}
$$

$$
\begin{aligned}
& \eta(k)=x(k+1)-x(k), \\
& V_{5}(k)=\left\{\begin{array}{c}
\sum_{i=k-\tau_{1}}^{k-1} x^{T}(i) Q_{5} x(i)+\left(\tau^{*}-\tau_{1}\right) \\
\times \sum_{i=-\tau^{*}} \sum_{j=k+i}^{k-\tau_{1}-1} \eta^{T}(j) Z_{2} \eta(j), \\
\tau_{1} \leq \tau(k) \leq \tau^{*} \\
\sum_{i=k-\tau_{2}}^{k-1} x^{T}(i) Q_{5} x(i)+\left(\tau_{2}-\tau^{*}\right) \\
\times \sum_{i=-\tau_{2}}^{-\tau^{*}-1} \sum_{j=k+i}^{k-1} \eta^{T}(j) Z_{2} \eta(j), \\
\tau^{*}<\tau(k) \leq \tau_{2},
\end{array}\right. \\
& V_{6}(k)=\left\{\begin{array}{lc}
\sum_{i=-d(k)}^{-1} \sum_{j=k+i}^{k-1} h^{T}(x(j)) R h(x(j)) \\
+\sum_{i=-d^{*}} \sum_{j=i+1}^{-d_{1}-1} \sum_{l=k+j}^{-1} h^{T}(x(l)) R h(x(l)), \\
\sum_{i=-d(k)}^{-1} \sum_{j=k+i}^{k-1} h^{T}(x(j)) R h(x(j)) \\
+\sum_{i=-d_{2}} \sum_{j=i+1}^{-1} \sum_{l=k+j}^{k-1} h^{T}(x(l)) R h(x(l)), \\
\end{array}\right.
\end{aligned}
$$

Calculating the difference of $V(k, x(k))$ and taking the mathematical expectation, by Lemma 6 , we have

$$
\begin{aligned}
& E \Delta V_{1}(k, x(k)) \\
&= E\left[x^{T}(k+1) P x(k+1)-x^{T}(k) P x(k)\right] \\
&= E\left[\eta^{T}(k) P \eta(k)+2 \eta^{T}(k) P x(k)\right] \\
& E \Delta V_{2}(k) \\
&=E\left[x^{T}(k) Q_{1} x(k)-x^{T}\left(k-\tau^{*}\right) Q_{1} x\left(k-\tau^{*}\right)\right. \\
&\left.\quad+x^{T}(k) Q_{2} x(k)-x^{T}\left(k-d^{*}\right) Q_{2} x\left(k-d^{*}\right)\right] \\
& E \Delta V_{3}(k) \\
&=E\left[\left(\sum_{i=k+1-\tau(k+1)}^{k}-\sum_{i=k-\tau(k)}^{k-1}\right) x^{T}(i) Q_{3} x(i)\right. \\
& \quad+\sum_{i=\tau_{1}}^{\tau_{2}-1}\left(\sum_{j=k-i+1}^{k}-\sum_{j=k-i}^{k-1}\right) x^{T}(j) Q_{3} x(j) \\
& \quad+\left(\sum_{i=k-\tau_{1}+1}^{k}-\sum_{i=k-\tau_{1}}^{k-1}\right) x^{T}(i) Q_{3} x(i) \\
& \quad+\sum_{-\tau_{1}+1}^{k}\left(\sum_{j=-\tau_{2}+1}^{k}-\sum_{j=k+i}^{k-1}\right) \\
&\left.\quad \times x^{T}(j) Q_{4} x(j)\right]
\end{aligned}
$$




$$
\begin{aligned}
& \leq E\left[\left(\sum_{i=k+1-\tau_{2}}^{k} x^{T}(i) Q_{3} x(i)-\sum_{i=k-\tau_{1}}^{k-1} x^{T}(i) Q_{3} x(i)\right)\right. \\
& +\sum_{i=\tau_{1}}^{\tau_{2}-1}\left(x^{T}(k) Q_{3} x(k)-x^{T}(k-i) Q_{3} x(k-i)\right) \\
& +\left(x^{T}(k) Q_{3} x(k)-x^{T}\left(k-\tau_{1}\right) Q_{3} x\left(k-\tau_{1}\right)\right) \\
& \psi(k)= \begin{cases}\sum_{i=k-\tau^{*}}^{k-\tau_{1}-1} \eta^{T}(i) Z_{2} \eta(i), & \tau_{1} \leq \tau(k) \leq \tau^{*}, \\
\sum_{i=k-\tau_{2}}^{k-\tau^{*}-1} \eta^{T}(i) Z_{2} \eta(i), & \tau^{*}<\tau(k) \leq \tau_{2} .\end{cases}
\end{aligned}
$$$$
+\sum_{i=-\tau_{2}+1}^{-\tau_{1}+1}\left(x^{T}(k) Q_{4} x(k)-x^{T}\right.
$$$$
\left.\left.\times(k+i-1) Q_{4} x(k+i-1)\right)\right]
$$$$
=E\left[x^{T}(k)\left[a\left(Q_{3}+Q_{4}\right)+Q_{3}\right] x(k)\right.
$$$$
-2 x^{T}\left(k-\tau_{1}\right) Q_{3} x\left(k-\tau_{1}\right)
$$$$
\left.-\sum_{i=k-\tau_{2}}^{k-\tau_{1}} x^{T}(i) Q_{4} x(i)\right]
$$$$
\leq E\left[x^{T}(k)\left[a\left(Q_{3}+Q_{4}\right)+Q_{3}\right] x(k)\right.
$$$$
\left.-x^{T}(k-\tau(k)) Q_{4} x(k-\tau(k))\right],
$$

$E \Delta V_{4}(k)$

$$
\begin{gathered}
=E\left[\tau^{*} \sum_{i=-\tau^{*}}^{-1}\left(\sum_{j=k+i+1}^{k}-\sum_{j=k+i}^{k-1}\right) \eta^{T}(j) Z_{1} \eta(j)\right] \\
=E\left[\tau^{* 2} \eta^{T}(k) Z_{1} \eta(k)-\tau^{*} \sum_{i=k-\tau^{*}}^{k-1} \eta^{T}(i) Z_{1} \eta(i)\right] \\
\leq E\left[\tau^{* 2} \eta^{T}(k) Z_{1} \eta(k)\right. \\
\left.-\sum_{i=k-\tau^{*}}^{k-1} \eta^{T}(i) Z_{1} \sum_{i=k-\tau^{*}}^{k-1} \eta(i)\right] .
\end{gathered}
$$

Note that

$$
\begin{aligned}
& -\sum_{i=k-\tau^{*}}^{k-1} \eta^{T}(i) Z_{1} \sum_{i=k-\tau^{*}}^{k-1} \eta(i) \\
& \quad=\left(\begin{array}{c}
x(k) \\
x\left(k-\tau^{*}\right)
\end{array}\right)^{T}\left(\begin{array}{cc}
-Z_{1} & Z_{1} \\
* & -Z_{1}
\end{array}\right)\left(\begin{array}{c}
x(k) \\
x\left(k-\tau^{*}\right)
\end{array}\right),
\end{aligned}
$$

$$
\begin{array}{r}
E \Delta V_{5}(k)=E\left\{x^{T}(k) Q_{5} x(k)-\theta^{T}(k) Q_{5} \theta(k)\right. \\
\left.+\tilde{\tau}^{2} \eta^{T}(k) Z_{2} \eta(k)-\tilde{\tau} \psi(k)\right\},
\end{array}
$$

When $\tau^{*}<\tau(k) \leq \tau_{2}$, it is easy to compute that

$$
\begin{aligned}
-\tilde{\tau} \psi & (k) \\
= & -\left[\left(\tau_{2}-\tau(k)\right)+\left(\tau(k)-\tau^{*}\right)\right] \\
& \times \sum_{i=k-\tau(k)}^{k-\tau^{*}-1} \eta^{T}(i) Z_{2} \eta(i) \\
& -\left[\left(\left(\tau_{2}-\tau(k)\right)+\left(\tau(k)-\tau^{*}\right)\right)\right] \\
& \times \sum_{i=k-\tau_{2}}^{k-\tau(k)-1} \eta^{T}(i) Z_{2} \eta(i) \\
\leq & \beta \sum_{i=k-\tau(k)}^{k-\tau^{*}-1} \eta^{T}(i) Z_{2} \sum_{i=k-\tau(k)}^{k-\tau^{*}-1} \eta(i) \\
& -\sum_{i=k-\tau(k)}^{k-\tau^{*}-1} \eta^{T}(i) Z_{2} \sum_{i=k-\tau(k)}^{k-\tau^{*}-1} \eta(i) \\
& \times \sum_{i=k-\tau(k)-1}^{k} \eta^{T}(i) Z_{2} \sum_{i=k-\tau_{2}}^{k-\tau(k)-1} \eta(i)-(1-\beta) \\
& \sum_{k-\tau(k)-1} \eta^{T}(i) Z_{2} \sum_{i=k-\tau_{2}}^{k-\tau(k)-1} \eta(i)
\end{aligned}
$$

$$
\begin{aligned}
-\widetilde{\tau} \psi(k) \leq & -(1-\beta) \sum_{i=k-\tau(k)}^{k-\tau_{1}-1} \eta^{T}(i) Z_{2} \sum_{i=k-\tau(k)}^{k-\tau_{1}-1} \eta(i) \\
& -\sum_{i=k-\tau(k)}^{k-\tau_{1}-1} \eta^{T}(i) Z_{2} \sum_{i=k-\tau(k)}^{k-\tau-1} \eta(i) \\
& -\sum_{i=k-\tau^{*}}^{k-\tau(k)-1} \eta^{T}(i) Z_{2} \sum_{i=k-\tau^{*}}^{k-\tau(k)-1} \eta(i) \\
& -\beta \sum_{i=k-\tau^{*}}^{k-\tau(k)-1} \eta^{T}(i) Z_{2} \sum_{i=k-\tau^{*}}^{k-\tau(k)-1} \eta(i) .
\end{aligned}
$$


From (20), (22), and (23), we have

$$
\begin{aligned}
E \Delta V_{5}(k)=E[ & x^{T}(k) Q_{5} x(k)-\theta^{T}(k) Q_{5} \theta(k) \\
& +\tilde{\tau}^{2} \eta^{T}(k) Z_{2} \eta(k)+\left(\begin{array}{c}
x(k-\tau(k)) \\
x\left(k-\tau^{*}\right) \\
\theta(k)
\end{array}\right) \\
& \times\left(\begin{array}{ccc}
-2 Z_{2} & Z_{2} & Z_{2} \\
* & -Z_{2} & 0 \\
* & * & -Z_{2}
\end{array}\right)\left(\begin{array}{c}
x(k-\tau(k)) \\
x\left(k-\tau^{*}\right) \\
\theta(k)
\end{array}\right) \\
& +\beta\left(\begin{array}{cc}
x(k-\tau(k)) \\
x\left(k-\tau^{*}\right)
\end{array}\right)^{T}\left(\begin{array}{cc}
-Z_{2} & Z_{2} \\
* & -Z_{2}
\end{array}\right) \\
& \times\left(\begin{array}{c}
x(k-\tau(k)) \\
x\left(k-\tau^{*}\right)
\end{array}\right)+\left(\begin{array}{cc}
1-\beta) \\
\end{array}\right. \\
& \times\left(\begin{array}{c}
x(k-\tau(k)) \\
\theta(k)
\end{array}\right)^{T}\left(\begin{array}{cc}
-Z_{2} & Z_{2} \\
* & -Z_{2}
\end{array}\right) \\
& \left.\times\left(\begin{array}{c}
x(k-\tau(k)) \\
\theta(k)
\end{array}\right)\right] .
\end{aligned}
$$

When $d_{1} \leq d(k) \leq d^{*}$, by Lemma 6 , it is easy to get

$$
\begin{aligned}
E \Delta V_{6}(k)=E[ & \left(\sum_{i=-d(k+1)}^{-1} \sum_{j=k+i+1}^{k}-\sum_{i=-d(k)}^{-1} \sum_{j=k+i}^{k-1}\right) \\
& \times h^{T}(x(j)) R h(x(j)) \\
& +\sum_{i=-d^{*}}^{-d_{1}-1} \sum_{j=i+1}^{-1}\left(\sum_{l=k+j+1}^{k}-\sum_{l=k+j}^{k-1}\right) \\
& \left.\times h^{T}(x(l)) R h(x(l))\right] \\
{\left[\begin{array}{l}
\sum_{i=-d^{*}} \\
\leq
\end{array}\right.} & \sum_{j=k+i+1}^{k-1} h^{T}(x(j)) R h(x(j)) \\
& -\sum_{i=-d(k)}^{-1} \sum_{j=k+i+1}^{k-1} h^{T}(x(j)) R h(x(j)) \\
& +\sum_{i=-d^{*}} \sum_{j=i+1}^{-1} h^{T}(x(k)) R h(x(k)) \\
& +\sum_{i=-d^{*}}^{-1} h^{T}(x(k)) R h(x(k)) \\
&
\end{aligned}
$$

$$
\begin{aligned}
\leq E\left[\frac{\left(d^{*}+d_{1}-1\right)\left(d^{*}-d_{1}\right)+2 d^{*}}{2}\right. \\
\quad \times h^{T}(x(k)) R h(x(k)) \\
\quad-\frac{1}{d^{*}}\left(\sum_{i=-d(k)}^{-1} h(x(k+i))\right)^{T} \\
\left.\quad \times R\left(\sum_{i=-d(k)}^{-1} h(x(k+i))\right)\right] .
\end{aligned}
$$

When $d^{*}<d(k) \leq d_{2}$, similarly we can have

$$
\begin{aligned}
& E \Delta V_{6}(k) \leq E\left[\frac{\left(d^{*}+d_{2}-1\right)\left(d_{1}-d^{*}\right)+2 d_{2}}{2}\right. \\
& \times h^{T}(x(k)) R h(x(k)) \\
&-\frac{1}{d_{2}}\left(\sum_{i=-d(k)}^{-1} h(x(k+i))\right)^{T} \\
&\left.\times R\left(\sum_{i=-d(k)}^{-1} h(x(k+i))\right)\right] .
\end{aligned}
$$

From (25) and (26), we have

$$
\begin{aligned}
E \Delta V_{6}(k) \leq E[ & b h^{T}(x(k)) R h(x(k)) \\
& -\frac{1}{c}\left(\sum_{i=-d(k)}^{-1} h(x(k+i))\right)^{T} \\
& \left.\times R\left(\sum_{i=-d(k)}^{-1} h(x(k+i))\right)\right] .
\end{aligned}
$$

From (7), it follows that

$$
\begin{aligned}
& \left(h_{i}(x(k))-\gamma_{i}^{+} x_{i}(k)\right) \\
& \quad \times\left(h_{i}(x(k))-\gamma_{i}^{-} x_{i}(k)\right) \leq 0, \quad i=1,2, \ldots, n,
\end{aligned}
$$

which are equivalent to

$$
\begin{aligned}
& \left(\begin{array}{c}
x(k) \\
h(x(k))
\end{array}\right)^{T}\left(\begin{array}{cc}
\gamma_{i}^{-} \gamma_{i}^{+} e_{i} e_{i}^{T} & -\frac{\gamma_{i}^{-}+\gamma_{i}^{+}}{2} e_{i} e_{i}^{T} \\
* & e_{i} e_{i}^{T}
\end{array}\right) \\
& \times\left(\begin{array}{c}
x(k) \\
h(x(k))
\end{array}\right) \leq 0,
\end{aligned}
$$

where $e_{i}$ denotes the unit column vector having one element on its $i$ th row and zeros elsewhere.

Then from (29), for any matrices $\Lambda=\operatorname{diag}\left\{\lambda_{1}, \lambda_{2}, \ldots\right.$, $\left.\lambda_{n}\right\}>0$, it follows that

$$
\left(\begin{array}{c}
x(k) \\
h(x(k))
\end{array}\right)^{T}\left(\begin{array}{cc}
-\Gamma_{1} \Lambda & \Gamma_{2} \Lambda \\
* & -\Lambda
\end{array}\right)\left(\begin{array}{c}
x(k) \\
h(x(k))
\end{array}\right) \geq 0 .
$$


Note that, by Lemma 7, we get

$$
\begin{gathered}
E\left[\eta^{T}(k) P \eta(k)+2 \eta^{T}(k) P x(k)+\tau^{* 2} \eta^{T}(k) Z_{1} \eta(k)\right. \\
\left.+\widetilde{\tau} \eta^{T}(k) Z_{2} \eta(k)\right] \\
=E\left[\eta^{T}(k)\left(P+\tau^{* 2} Z_{1}+\tilde{\tau} Z_{2}\right) \eta(k)+2 \eta^{T}(k) P x(k)\right] \\
=E\left[x^{T}(k+1)\left(P+\tau^{* 2} Z_{1}+\tilde{\tau} Z_{2}\right) x(k+1)\right. \\
\quad-2 x^{T}(k+1)\left(\tau^{* 2} Z_{1}+\tilde{\tau} Z_{2}\right) x(k) \\
\left.\quad+x^{T}(k)\left(\tau^{* 2} Z_{1}+\tilde{\tau} Z_{2}-P\right) x(k)\right] \\
\leq E\left[x^{T}(k+1)\left(P+2 \tau^{* 2} Z_{1}+2 \widetilde{\tau} Z_{2}\right) x(k+1)\right. \\
\left.\quad+x^{T}(k)\left(2 \tau^{* 2} Z_{1}+2 \tilde{\tau} Z_{2}-P\right) x(k)\right] \\
\leq E\left[x^{T}(k+1) \lambda^{*} I x(k+1)+x^{T}(k)\right. \\
\left.\quad \times\left(2 \tau^{* 2} Z_{1}+2 \widetilde{\tau} Z_{2}-P\right) x(k)\right]
\end{gathered}
$$$$
=E\{[(A+C K) x(k)+B x(k-\tau(k))
$$$$
+D \sum_{i=-d(k)}^{-1} h(x(k+i))
$$$$
+f(k, x(k), x(k-\tau(k)))+C r(k)]^{T}
$$$$
\times \lambda^{*} I[(A+C K) x(k)+B x(k-\tau(k))
$$$$
+D \sum_{i=-d(k)}^{-1} h(x(k+i))
$$$$
+f(k, x(k), x(k-\tau(k)))+C r(k)]
$$$$
+[G x(k)+H x(k-\tau(k))]^{T}
$$$$
\times \lambda^{*} I[G x(k)+H x(k-\tau(k))]
$$$$
\left.+x^{T}(k)\left(2 \tau^{* 2} Z_{1}+2 \widetilde{\tau} Z_{2}-P\right) x(k)\right\}
$$$$
\leq E\{[(A+C K) x(k)+B x(k-\tau(k))
$$$$
+D \sum_{i=-d(k)}^{-1} h(x(k+i))
$$$$
+f(k, x(k), x(k-\tau(k)))]^{T} \lambda^{*} I
$$$$
\times[(A+C K) x(k)+B x(k-\tau(k))
$$$$
\left.+D \sum_{i=-d(k)}^{-1} h(x(k+i))+f(k, x(k), x(k-\tau(k)))\right]
$$

$$
\begin{aligned}
& +[G x(k)+H x(k-\tau(k))]^{T} \lambda^{*} I \\
& \times[G x(k)+H x(k-\tau(k))] \\
& +x^{T}(k)\left(2 \tau^{* 2} Z_{1}+2 \widetilde{\tau} Z_{2}-P\right) x(k) \\
& +\lambda^{*} x^{T}(A+C K)^{T}(A+C K) x(k) \\
& +\lambda^{*} x^{T}(k-\tau(k)) B^{T} B x(k-\tau(k)) \\
& +\lambda^{*}\left(\sum_{i=-d(k)}^{-1} h(x(k+i))\right)^{T} D^{T} D \\
& \times\left(\sum_{i=-d(k)}^{-1} h(x(k+i))\right) \\
& \left.+5 \lambda^{*}\|C\|^{2}\|r\|_{\infty}^{2}\right\} .
\end{aligned}
$$

Then from (18) to (31), we have

$$
\begin{aligned}
E \Delta V(k) \leq E & \left\{\xi ^ { T } ( k ) \left[\Xi^{\prime}+(\sqrt{2} X, 0,0,0,0,0,0,0)^{T} \frac{1}{\lambda^{*}}\right.\right. \\
& \times(\sqrt{2} X, 0,0,0,0,0,0,0)] \xi(k)\} \\
& +\rho\|r\|_{\infty}^{2},
\end{aligned}
$$

where

$$
\begin{gathered}
\Xi_{i j}^{\prime}=\Xi_{i j}, \quad i, j=1,2, \ldots, 8, \quad \rho=5 \lambda^{*}\|D\|^{2}, \\
\xi^{T}(k)=\left[x^{T}(k), x^{T}(k-\tau(k)), x\left(k-\tau^{*}\right), x\left(k-d^{*}\right),\right. \\
\left.\theta^{T}(k), h^{T}(x(k)), \sum_{-d(k)}^{-1} h^{T}(x(k+i)), f^{T}\right] .
\end{gathered}
$$

If the LMI (14) holds, by using Lemma 8, it follows that there exists a sufficient small positive $\varepsilon>0$, such that

$$
E \Delta V(k) \leq-\varepsilon E\|x(k)\|^{2}+\rho\|r\|_{\infty}^{2} .
$$

It is easy to derive that

$$
\begin{aligned}
E V(k) \leq & \mu_{1} E\|x(k)\|^{2}+\mu_{2} \sum_{i=k-\tau_{2}}^{k-1} E\|x(i)\|^{2} \\
& +\mu_{3} \sum_{i=k-d_{2}}^{k-1} E\|x(i)\|^{2},
\end{aligned}
$$


with

$$
\begin{aligned}
\mu_{1}= & \lambda_{\max }(P), \\
\mu_{2}= & \lambda_{\max }\left(Q_{1}\right)+(a+1) \lambda_{\max }\left(Q_{3}\right) \\
& +2 \lambda_{\max }\left(Q_{4}\right)+4 \tau^{* 2} \lambda_{\max }\left(Z_{1}\right) \\
& +4\left(\tau_{2}-\tau_{1}\right)^{2} \lambda_{\max }\left(Z_{2}\right), \\
\mu_{3}= & \lambda_{\max }\left(Q_{2}\right)+\left[d_{2}+\left(d_{2}-d_{1}\right)\right. \\
& \left.\times\left(d_{2}-d_{1}\right)\right] \\
& \times\|\Gamma\|^{2} \lambda_{\max }(R) .
\end{aligned}
$$

For any $\theta>1$, it follows from (34) and (35) that

$$
\begin{aligned}
& E\left[\theta^{j+1} V(j+1)-\theta^{j} V(j)\right] \\
& =\theta^{j+1} E \Delta V(j)+\theta^{j}(\theta-1) E V(j) \\
& \leq \theta^{j}\left[\left(-\varepsilon \theta+(\theta-1) \mu_{1}\right) E\|x(j)\|^{2}\right. \\
& +(\theta-1) \mu_{2} \sum_{i=j-\tau_{2}}^{j-1} E\|x(i)\|^{2}+\rho \theta\|r\|_{\infty}^{2} \\
& \left.+(\theta-1) \mu_{3} \sum_{i=j-d_{2}}^{j-1} E\|x(i)\|^{2}\right] .
\end{aligned}
$$

Summing up both sides of (37) from 0 to $k-1$, we can obtain

$$
\begin{aligned}
& \theta^{k} E V(k)-E V(0) \\
& \leq\left(\mu_{1}(\theta-1)-\varepsilon \theta\right) \sum_{j=0}^{k-1} \theta^{j} E\|x(j)\|^{2} \\
& \quad+\mu_{2}(\theta-1) \sum_{j=0}^{k-1} \sum_{i=j-\tau_{2}}^{j-1} \theta^{j} E\|x(i)\|^{2} \\
& \quad+\mu_{3}(\theta-1) \sum_{j=0}^{k-1} \sum_{i=j-d_{2}}^{j-1} \theta^{j} E\|x(i)\|^{2} \\
& \quad+\rho \sum_{j=0}^{k-1} \theta^{j+1}\|r\|_{\infty}^{2} .
\end{aligned}
$$

Also it is easy to compute that

$$
\begin{aligned}
& \sum_{j=0}^{k-1} \sum_{i=j-\tau_{2}}^{j-1} \theta^{j} E\|x(i)\|^{2} \\
& \leq\left(\sum_{i=-\tau_{2}}^{-1} \sum_{j=0}^{i+\tau_{2}}+\sum_{i=0}^{k-1-\tau_{2}} \sum_{j=i+1}^{i+\tau_{2}}+\sum_{i=k-\tau_{2}}^{k-1} \sum_{j=i+1}^{k-1}\right) \\
& \quad \times \theta^{j} E\|x(i)\|^{2}
\end{aligned}
$$

$\leq \tau_{2} \theta^{\tau_{2}} \sup _{s \in\left[-\tau_{2}, 0\right]} E\|x(s)\|^{2}+\tau_{2} \theta^{\tau_{2}} \sum_{i=0}^{k-1} \theta^{i} E\|x(i)\|^{2}$,

$\sum_{j=0}^{k-1} \sum_{i=j-d_{2}}^{j-1} \theta^{j} E\|x(i)\|^{2}$

$\leq\left(\sum_{i=-d_{2}}^{-1} \sum_{j=0}^{i+d_{2}}+\sum_{i=0}^{k-1-d_{2}} \sum_{j=i+1}^{i+d_{2}}+\sum_{i=k-d_{2}}^{k-1} \sum_{j=i+1}^{k-1}\right)$

$\times \theta^{j} E\|x(i)\|^{2}$

$\leq d_{2} \theta^{d_{2}} \sup _{s \in\left[-d_{2}, 0\right]} E\|x(s)\|^{2}+d_{2} \theta^{d_{2}}$

$\times \sum_{i=0}^{k-1} \theta^{i} E\|x(i)\|^{2}$

Substituting (39) into (38) leads to

$$
\begin{aligned}
& \theta^{k} E V(k)-E V(0) \\
& \leq \eta_{1}(\theta) \sup _{s \in\left[-\tau_{2}, 0\right]} E\|x(s)\|^{2}+\rho \sum_{j=0}^{k-1} \theta^{j+1}\|r\|_{\infty}^{2} \\
& \quad+\eta_{2}(\theta) \sum_{i=0}^{k-1} \theta^{i} E\|x(i)\|^{2}+\eta_{3}(\theta) \sum_{i=0}^{k-1} \theta^{i} E\|x(i)\|^{2},
\end{aligned}
$$

where $\eta_{1}(\theta)=\mu_{2}(\theta-1) \tau_{2} \theta^{\tau_{2}}+\mu_{3}(\theta-1) d_{2} \theta^{d_{2}}, \eta_{2}(\theta)=\mu_{2}(\theta-$ 1) $\tau_{2} \theta^{\tau_{2}}+\mu_{1}(\theta-1)-\varepsilon \theta, \eta_{3}(\theta)=\mu_{3}(\theta-1) d_{2} \theta^{d_{2}}+\mu_{1}(\theta-1)-\varepsilon \theta$.

Since $\eta_{2}(1)<0, \eta_{3}(1)<0$, there must exist a positive $\theta_{0}>1$ such that $\eta_{2}\left(\theta_{0}\right)<0, \eta_{3}\left(\theta_{0}\right)<0$. Then we have

$E V(k)$

$$
\begin{aligned}
& \leq \eta_{1}\left(\theta_{0}\right)\left(\frac{1}{\theta_{0}}\right)^{k} \sup _{s \in\left[-\tau_{2}, 0\right]} E\|x(s)\|^{2} \\
& +\left(\frac{1}{\theta_{0}}\right)^{k} E V(0)+\rho \sum_{j=0}^{k-1} \frac{1}{\theta_{0}^{k-j-1}}\|r\|_{\infty}^{2} \\
& \quad+\eta_{2}\left(\theta_{0}\right) \sum_{i=0}^{k-1} \frac{1}{\theta_{0}^{k-i}} E\|x(i)\|^{2}+\eta_{3}\left(\theta_{0}\right) \sum_{i=0}^{k-1} \frac{1}{\theta_{0}^{k-i}} E\|x(i)\|^{2} \\
& \leq\left(\eta_{1}\left(\theta_{0}\right)+\mu_{1}+\mu_{2} \tau_{2}+\mu_{3} d_{2}\right) \\
& \quad \times \sup _{s \in\left[-\max _{\left.\left\{\tau_{2}, d_{2}\right\}, 0\right]} E\|x(s)\|^{2}+\frac{\rho}{\theta_{0}-1}\|r\|_{\infty}^{2} .\right.}
\end{aligned}
$$

On the other hand, by (16) we can get

$$
E V(k) \geq \lambda_{\min }(P) E\|x(k)\|^{2} .
$$

Combining (41) with (42), we have

$$
\begin{aligned}
E\|x(k)\|^{2} \leq & \frac{\eta_{1}\left(\theta_{0}\right)+\mu_{1}+\mu_{2} \tau_{2}+\mu_{3} d_{2}}{\lambda_{\min }(P)} \\
& \times \sup _{s \in\left[-\max \left\{\tau_{2}, d_{2}\right\}, 0\right]} E\|x(s)\|^{2} \\
& +\frac{1}{\lambda_{\min }(P)} \frac{\rho}{\theta_{0}-1}\|r\|_{\infty}^{2} .
\end{aligned}
$$


Thus,

$$
\mathbb{E}\|y(k)\|^{2} \leq\|M\|^{2} E\|x(k)\|^{2} \leq N_{1}+N_{2}\|r\|_{\infty}^{2},
$$

where $N_{1}=\|M\|^{2}\left(\left(\eta_{1}\left(\theta_{0}\right)+\mu_{1}+\mu_{2} \tau_{2}+\mu_{3} d_{2}\right) / \lambda_{\min }(P)\right)$ $\sup _{s \in\left[-\max \left\{\tau_{2}, d_{2}\right\}, 0\right]} E\|x(s)\|^{2}, \quad N_{2}=\left(1 / \lambda_{\min }(P)\right)\left(\rho /\left(\theta_{0}-\right.\right.$ 1)) $\|M\|^{2}$. By Definition 5 , the nonlinear discrete-time stochastic control system (1) is BIBO stability in mean square. This completes the proof.

If the stochastic term $\omega(K)$ is removed in (1), then the following results can be obtained.

Theorem 10. For given positive integers $\tau_{1}, \tau_{2}, d_{1}$, and $d_{2}$, under Assumption 2, the nonlinear discrete-time stochastic control system (1) with the controller (6) is BIBO stabilization in mean square, if there exist symmetric positive-definite matrix $P, R, Q_{i}, i=1,2, \ldots, 5, Z_{1}, Z_{2}$, and $X$ with appropriate dimensional positive-definite diagonal matrices $\Lambda$ and two positive constants $\zeta$ and $\lambda^{*}$ such that the following two LMIs hold:

$$
P+2\left(\tau^{* 2} Z_{1}+\widetilde{\tau}^{2} Z_{2}\right) \leq \lambda^{*} I
$$

$\Xi$

$$
=\left(\begin{array}{ccccccccc}
\Xi_{11} & \Xi_{12} & Z_{1} & 0 & 0 & \Gamma_{2} \Lambda & \Xi_{17} & \Xi_{18} & \sqrt{2} X \\
* & \Xi_{22} & \Xi_{23} & 0 & \Xi_{25} & 0 & B^{T} D & B^{T} & 0 \\
* & * & \Xi_{33} & 0 & 0 & 0 & 0 & 0 & 0 \\
* & * & * & -Q_{2} & 0 & 0 & 0 & 0 & 0 \\
* & * & * & * & \Xi_{55} & 0 & 0 & 0 & 0 \\
* & * & * & * & * & \Xi_{66} & 0 & 0 & 0 \\
* & * & * & * & * & * & \Xi_{77} & \lambda^{*} D^{T} & 0 \\
* & * & * & * & * & * & * & -\zeta I & 0 \\
* & * & * & * & * & * & * & * & -\lambda^{*} I
\end{array}\right)
$$

$\leq 0$,

where

$$
\begin{aligned}
\Xi_{11}= & Q_{1}+Q_{2}+Q_{3}+a\left(Q_{3}+Q_{4}\right)-Z_{1} \\
& +Q_{5}-\Gamma_{1} \Lambda+\zeta I+2 \tau^{* 2} Z_{1}+2 \tau^{2} Z_{2} \\
& +2 \lambda^{*} \alpha_{1} I+\alpha_{1} \zeta I-P+2 \lambda^{*} A^{T} A \\
& -2 A^{T} X-2 X^{T} A, \\
\Xi_{22}= & 2 \lambda^{*} B^{T} B-Q_{4}-2 Z_{2}-\beta Z_{2} \\
& +2 \lambda^{*} \alpha_{2} I+\alpha_{2} \zeta I-(1-\beta) Z_{2},
\end{aligned}
$$

$$
\begin{aligned}
& \Xi_{12}=\lambda^{*} A^{T} B-X^{T} B, \\
& \Xi_{17}=\lambda^{*} A^{T} D-X^{T} D, \\
& \Xi_{18}=\lambda^{*} A^{T}-X^{T}, \\
& \Xi_{25}=Z_{2}+(1-\beta) Z_{2}, \\
& \Xi_{23}=Z_{2}+\beta Z_{2}, \\
& \Xi_{33}=-Q_{1}-Z_{1}-Z_{2}-\beta Z_{2}, \\
& \Xi_{55}=-Q_{5}-Z_{2}-(1-\beta) Z_{2}, \\
& \Xi_{66}=b R-\Lambda, \\
& \Xi_{77}=2 \lambda^{*} D^{T} D-\frac{1}{c} R, \\
& X=-\lambda^{*} C K .
\end{aligned}
$$

Proof. The proof is straightforward and hence omitted.

Corollary 11. System (1) is also stabilization in mean square when all the conditions in Theorems 9 and 10 are satisfied, if the bounded input $r(t)=0$ in (6).

Remark 12. In this paper, a novel $\mathrm{BIBO}$ stability criterion for system (1) is derived by checking the variation of derivatives of the Lyapunov-Krasovskii functionals for each subinterval. It is different from [27-32], which checked the variation of the Lyapunov functional in the whole variation interval of the delay.

Remark 13. The BIBO stabilization criteria for discrete-time systems have been investigated in the recently reported paper [28]. However, the stochastic disturbances and nonlinear perturbations have not been taken into account in the control systems. In [28], the time delay is constant time, which is a special case of this paper when $\tau_{1}=\tau_{2}$.

Remark 14. The mean square stabilization conditions in Theorem 9 in this paper depend on the time-delays upper bounds and the lower bounds, time-delays interval, and timedelay interval segmentation point and relate to the delays themselves.

Remark 15. In [33], the time-delay interval is divided into two equal subintervals; the interval segmentation point is midpoint. In this paper, the time-delay interval is divided into two any subintervals; the interval segmentation point is any point in the time-delay interval.

\section{An Example}

In this section, a numerical example will be presented to show the validity of the main results derived in Section 3. 
TABLE 1: For $\tau(k)=1,2,3,4,5, \beta$.

\begin{tabular}{lllccc}
\hline$\tau(k)$ & 1 & 2 & 3 & 4 & 5 \\
$\beta$ & 0 & 1 & $1 / 2$ & $1 / 2$ & 0 \\
\hline
\end{tabular}

Example 1. As a simple application of Theorem 9, consider the stochastic control system (1) with the control law (6); the parameters are given by

$$
\begin{aligned}
& A=\left(\begin{array}{cc}
-0.1 & 0 \\
0.1 & -0.2
\end{array}\right), \quad B=\left(\begin{array}{cc}
-0.1 & 0.1 \\
-0.1 & 0.1
\end{array}\right), \\
& C=\left(\begin{array}{cc}
0.1 & 0.1 \\
0.5 & 0.3
\end{array}\right), \quad D=\left(\begin{array}{cc}
0.1 & 0.1 \\
0 & 0.2
\end{array}\right),
\end{aligned}
$$

$G=0.001 I, H=0.02 I, f=[0.1 x(k), \sqrt{0.2} x(k-\tau(k))]^{T}$, $h_{1}(s)=\sin (0.2 s)-0.6 \cos (s), h_{2}(s)=\tanh (-0.4 s), \tau_{1}=1$, $\tau_{2}=5, d_{1}=2, d_{2}=7$.

It is easy to verify that $a=5, \tau^{*}=3, d^{*}=4, \widetilde{\tau}=2$, and

$$
\begin{aligned}
& \Gamma_{1}=\left(\begin{array}{cc}
-0.64 & 0 \\
0 & 0
\end{array}\right), \quad \Gamma_{2}=\left(\begin{array}{cc}
0 & 0 \\
0 & -0.2
\end{array}\right), \\
& \tau^{*}=\frac{\tau_{1}+\tau_{2}}{2}-\frac{\min \left\{(-1)^{\tau_{1}+\tau_{2}}, 0\right\}}{2}, \\
& d^{*}=\frac{\tau_{1}+\tau_{2}}{2}+\frac{\min \left\{(-1)^{\tau_{1}+\tau_{2}}, 0\right\}}{2}, \\
& b= \begin{cases}9, & d_{1} \leq d(k) \leq d^{*}, \\
20, & d^{*}<d(k) \leq d_{2},\end{cases} \\
& c= \begin{cases}4, & d_{1} \leq d(k) \leq d^{*}, \\
7, & d^{*}<d(k) \leq d_{2} .\end{cases}
\end{aligned}
$$

Meanwhile, the corresponding values of $\beta$ for various $\tau(k)$ are listed in Table 1.

By using the MATLAB LMI Toolbox, we solve LMIs (13), (14) and obtain six groups of feasible solutions; we list one case as follows.

$$
\text { When } \beta=1, b=9, c=4 \text {, }
$$

$$
\begin{aligned}
& P=\left(\begin{array}{cc}
145.0684 & -3.2651 \\
-3.2651 & 147.7799
\end{array}\right), \quad Z_{1}=\left(\begin{array}{ll}
0.0478 & 0.0298 \\
0.0298 & 0.0230
\end{array}\right), \\
& Z_{2}=\left(\begin{array}{ll}
0.3443 & 0.2142 \\
0.2142 & 0.1663
\end{array}\right), \quad Q_{1}=\left(\begin{array}{ll}
2.8401 & 1.7808 \\
1.7808 & 1.3624
\end{array}\right), \\
& Q_{2}=\left(\begin{array}{ll}
3.7464 & 2.3462 \\
2.3462 & 1.7985
\end{array}\right), \quad Q_{3}=\left(\begin{array}{ll}
0.2731 & 0.1713 \\
0.1713 & 0.1309
\end{array}\right) \text {, } \\
& Q_{4}=\left(\begin{array}{rr}
15.3027 & -6.8645 \\
-6.8645 & 15.5746
\end{array}\right), \quad Q_{5}=\left(\begin{array}{ll}
3.1500 & 1.9740 \\
1.9740 & 1.5118
\end{array}\right), \\
& R=\left(\begin{array}{rr}
128.2646 & -92.1100 \\
-92.1100 & 239.0910
\end{array}\right), \quad N=\left(\begin{array}{cc}
0.4512 & 0 \\
0 & 34.3341
\end{array}\right) \text {, } \\
& X=\left(\begin{array}{cc}
13.3495 & 3.1121 \\
3.1121 & 0.7692
\end{array}\right), \quad K=\left(\begin{array}{cc}
-1.4212 & -0.3303 \\
0.5268 & 0.1218
\end{array}\right) \text {, }
\end{aligned}
$$

and $\zeta=82.0259, \lambda^{*}=150.2996$.

The system (1) exhibits stabilization in mean square behavior as shown in Figure 1.


Figure 1

\section{Conclusions}

In this paper, we have derived some conditions for the BIBO stabilization in mean square for a class of discretetime stochastic control systems with mixed time-varying delays. The results have been obtained by constructing a novel Lyapunov-Krasovskii function. The conditions are expressed in the forms of linear matrix inequalities, which can be easily checked by using MATLAB LMI Toolbox. A numerical example is given to illustrate the validity of the obtained results.

\section{Acknowledgments}

The work of Xia Zhou is supported by the National Natural Science Foundation of China (no. 11226140) and the Anhui Provincial Colleges and Universities Natural Science Foundation (no. KJ2013Z267). The work of Yong Ren is supported by the National Natural Science Foundation of China (nos. 10901003 and 11126238), the Distinguished Young Scholars of Anhui Province (no. 1108085J08), the Key Project of Chinese Ministry of Education (no. 211077), and the Anhui Provincial Natural Science Foundation (no. 10040606Q30).

\section{References}

[1] R. Sakthivel, K. Mathiyalagan, and S. M. Anthoni, "Robust stability and control for uncertain neutral time delay systems," International Journal of Control, vol. 85, no. 4, pp. 373-383, 2012.

[2] S. Lakshmanan, J. H. Park, D. H. Ji, H. Y. Jung, and G. Nagamani, "State estimation of neural networks with time-varying delays and Markovian jumping parameter based on passivity theory," Nonlinear Dynamics, vol. 70, no. 2, pp. 1421-1434, 2012.

[3] R. Sakthivel, K. Mathiyalagan, and S. Marshal Anthoni, "Robust $H_{\infty}$ control for uncertain discrete-time stochastic neural networks with time-varying delays," IET Control Theory \& Applications, vol. 6, no. 9, pp. 1220-1228, 2012.

[4] P. Balasubramaniam, S. Lakshmanan, and A. Manivannan, "Robust stability analysis for Markovian jumping interval neural networks with discrete and distributed time-varying delays," Chaos, Solitons and Fractals, vol. 45, no. 4, pp. 483-495, 2012. 
[5] K. Mathiyalagan, R. Sakthivel, and S. Marshal Anthoni, "Exponential stability result for discrete-time stochastic fuzzy uncertain neural networks," Physics Letters A, vol. 376, no. 8-9, pp. 901-912, 2012.

[6] A. Arunkumar, R. Sakthivel, K. Mathiyalagan, and S. Marshal Anthoni, "Robust stability criteria for discrete-time switched neural networks with various activation functions," Applied Mathematics and Computation, vol. 218, no. 22, pp. 10803-10816, 2012.

[7] S. Lakshmanan and P. Balasubramaniam, "New results of robust stability analysis for neutral-type neural networks with timevarying delays and Markovian jumping parameters," Canadian Journal of Physics, vol. 89, no. 8, pp. 827-840, 2011.

[8] P. Vadivel, R. Sakthivel, K. Mathiyalagan, and P. Thangaraj, "Robust stabilization of nonlinear uncertain Takagi-Sugeno fuzzy systems by $\mathrm{H}$ control," IET Control Theory and Applications, vol. 6, pp. 2556-2566, 2012.

[9] Z. Liu, S. Lü, S. Zhong, and M. Ye, "Improved robust stability criteria of uncertain neutral systems with mixed delays," Abstract and Applied Analysis, Article ID 294845, 18 pages, 2009.

[10] F. Qiu, B. Cui, and Y. Ji, "Delay-dividing approach for absolute stability of Lurie control system with mixed delays," Nonlinear Analysis: Real World Applications, vol. 11, no. 4, pp. 3110-3120, 2010.

[11] X. Wang, Q. Guo, and D. Xu, "Exponential p-stability of impulsive stochastic Cohen-Grossberg neural networks with mixed delays," Mathematics and Computers in Simulation, vol. 79, no. 5, pp. 1698-1710, 2009.

[12] W. Zhou, H. Lu, and C. Duan, "Exponential stability of hybrid stochastic neural networks with mixed time delays and nonlinearity," Neurocomputing, vol. 72, no. 13-15, pp. 3357-3365, 2009.

[13] Y. Zhang, S. Xu, and Z. Zeng, "Novel robust stability criteria of discrete-time stochastic recurrent neural networks with time delay," Neurocomputing, vol. 72, no. 13-15, pp. 3343-3351, 2009.

[14] C. Peng and Y.-C. Tian, "Delay-dependent robust stability criteria for uncertain systems with interval time-varying delay," Journal of Computational and Applied Mathematics, vol. 214, no. 2, pp. 480-494, 2008.

[15] Z. Wu, H. Su, J. Chu, and W. Zhou, "Improved result on stability analysis of discrete stochastic neural networks with time delay," Physics Letters A, vol. 373, no. 17, pp. 1546-1552, 2009.

[16] R. Sakthivel, S. Santra, and K. Mathiyalagan, "Admissibility analysis and control synthesis for descriptor systems with random abrupt changes," Applied Mathematics and Computation, vol. 219, no. 18, pp. 9717-9730, 2013.

[17] Y. Liu, Z. Wang, and X. Liu, "State estimation for discretetime Markovian jumping neural networks with mixed modedependent delays," Physics Letters A, vol. 372, no. 48, pp. 71477155, 2008.

[18] Y. Tang, J.-A. Fang, M. Xia, and D. Yu, "Delay-distributiondependent stability of stochastic discrete-time neural networks with randomly mixed time-varying delays," Neurocomputing, vol. 72, no. 16-18, pp. 3830-3838, 2009.

[19] Y. Liu, Z. Wang, and X. Liu, "Asymptotic stability for neural networks with mixed time-delays: the discrete-time case," Neural Networks, vol. 22, no. 1, pp. 67-74, 2009.

[20] P. Li and S.-m. Zhong, "BIBO stabilization for system with multiple mixed delays and nonlinear perturbations," Applied Mathematics and Computation, vol. 196, no. 1, pp. 207-213, 2008.

[21] T. Bose and M. Q. Chen, "BIBO stability of the discrete bilinear system,” Digital Signal Processing, vol. 5, no. 3, pp. 160-166, 1995.
[22] S. M. Zhong and Y. Q. H. Uang, "BIBO stabilization of nonlinear system with time-delay," Journal of University of Electronic Science and Technology of China, vol. 32, no. 4, pp. 655-657, 2000.

[23] K. C. Cao, S. M. Zhong, and B. S. Liu, "BIBO and robust stabilization for system with time-delay and nonlinear perturbations," Journal of University of Electronic Science and Technology of China, vol. 32, no. 6, pp. 787-789, 2003.

[24] J. R. Partington and C. Bonnet, " $H_{\infty}$ and BIBO stabilization of delay systems of neutral type," Systems \& Control Letters, vol. 52, no. 3-4, pp. 283-288, 2004.

[25] A. T. Tomerlin and W. W. Edmonson, "BIBO stability of d-dimensional filters," Multidimensional Systems and Signal Processing, vol. 13, no. 3, pp. 333-340, 2002.

[26] Y. Q. Huang, W. Zeng, and S. M. Zhong, "BIBO stabukuty of continuous time systems," Journal of University of Electronic Science and Technology of China, vol. 3, no. 2, pp. 178-181, 2005.

[27] P. Li and S.-m. Zhong, "BIBO stabilization of time-delayed system with nonlinear perturbation," Applied Mathematics and Computation, vol. 195, no. 1, pp. 264-269, 2008.

[28] Z. X. Liu, S. Lv, and S. M. Zhong, "Augmented Lyapunov method for BIBO stabilization of discrete system," Journal of Mathematics Research, vol. 2, pp. 116-122, 2011.

[29] P. Li and S.-M. Zhong, "BIBO stabilization of piecewise switched linear systems with delays and nonlinear perturbations," Applied Mathematics and Computation, vol. 213, no. 2, pp. 405-410, 2009.

[30] P. Li, S.-m. Zhong, and J.-z. Cui, "Delay-dependent robust BIBO stabilization of uncertain system via LMI approach," Chaos, Solitons and Fractals, vol. 40, no. 2, pp. 1021-1028, 2009.

[31] Y. Fu and X. Liao, "BIBO stabilization of stochastic delay systems with uncertainty," Institute of Electrical and Electronics Engineers, vol. 48, no. 1, pp. 133-138, 2003.

[32] X. Zhou and S. Zhong, "Riccati equations and delay-dependent BIBO stabilization of stochastic systems with mixed delays and nonlinear perturbations," Advances in Difference Equations, Article ID 494607, 14 pages, 2010.

[33] D. Yue, "Robust stabilization of uncertain systems with unknown input delay," Automatica, vol. 40, no. 2, pp. 331-336, 2004. 


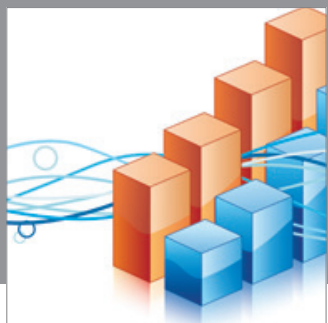

Advances in

Operations Research

mansans

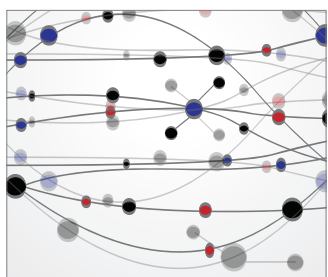

The Scientific World Journal
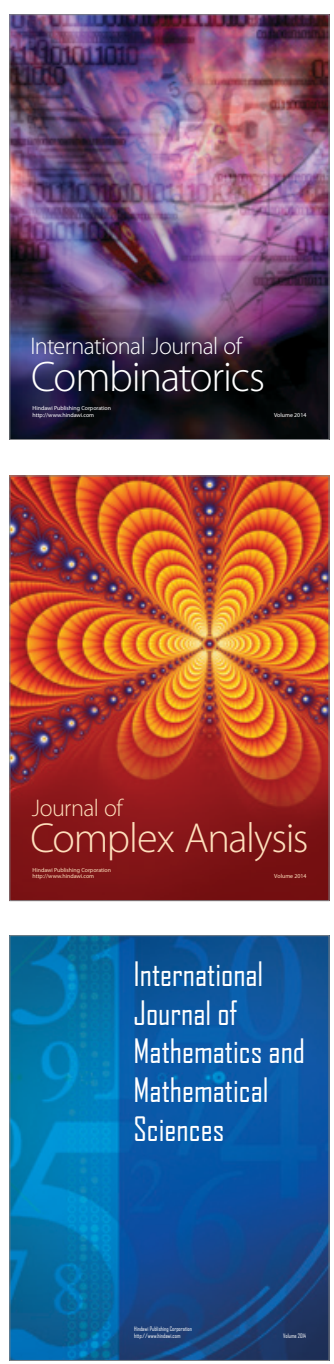


Submit your manuscripts at http://www.hindawi.com
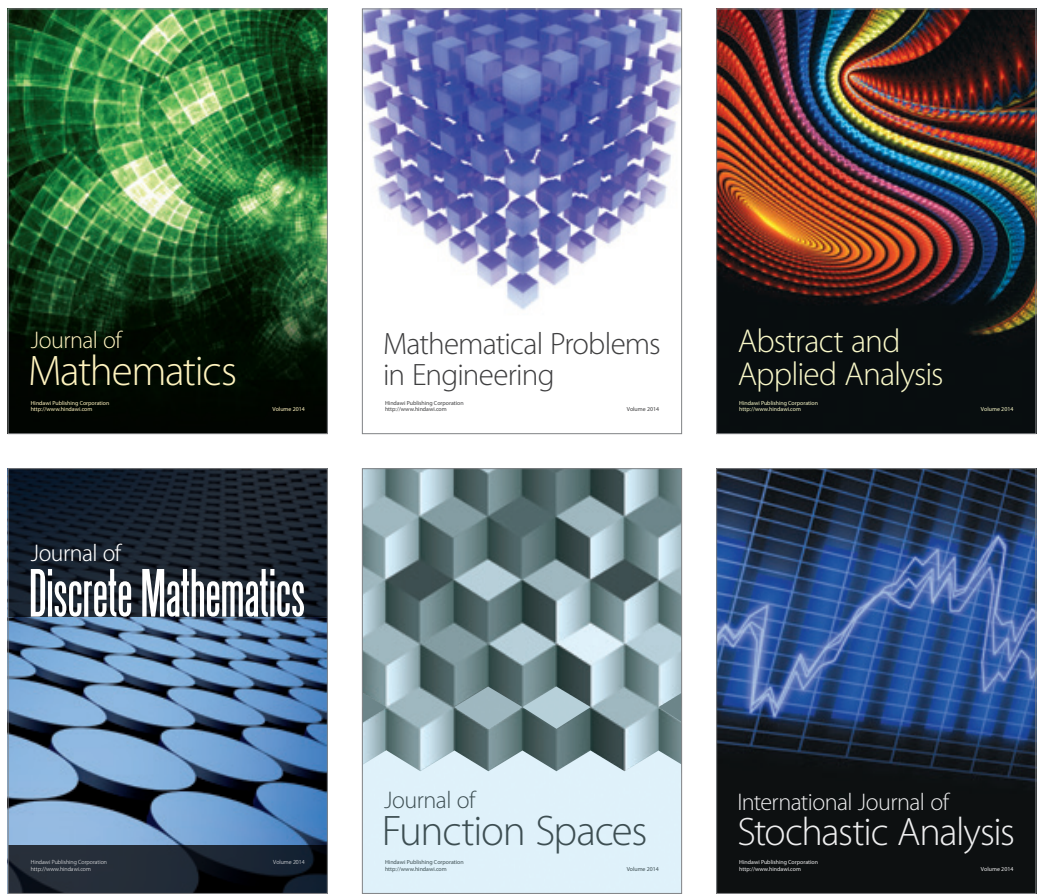

Journal of

Function Spaces

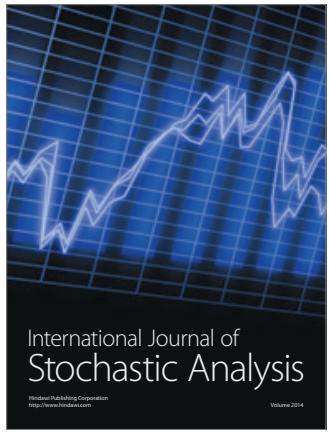

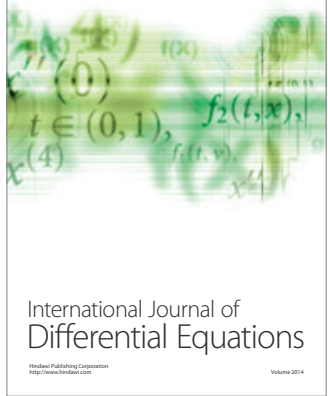
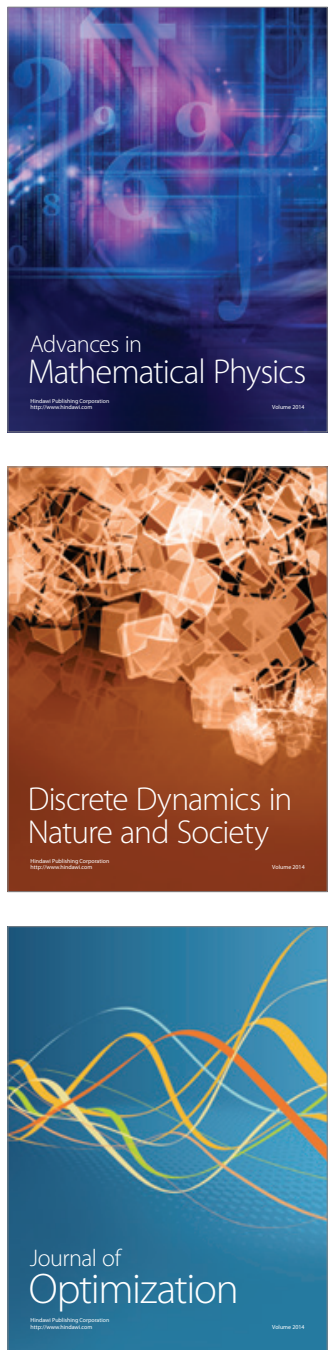